\title{
Quantitative Analysis of KH903 from Human Serum by Enzyme-Linked Immunosorbent Assay and its Application in a Pharmacokinetic Study
}

\author{
Bing Tian Bi ${ }^{1,2 \#}$, Ben Y. Zou ${ }^{1,3 \#}$, Min J. Zhao ${ }^{1,2}$, Wen L. Zhu ${ }^{4}$, Xiao Ke ${ }^{4}$, Li T. Deng', ${ }^{1,2}$ Hai Liao ${ }^{1,2}$, Jing \\ Zhan $^{1,2}$, Kun Y. Feng ${ }^{1,2}$ and Su Li ${ }^{1,2 *}$ \\ ${ }^{1}$ Sun Yat-sen University Cancer Center, State Key Laboratory of Oncology in South China, Collaborative Innovation Center of Cancer Medicine, Guangzhou \\ 510060, People's Republic of China \\ ${ }^{2}$ Department of Clinical Research Center, Cancer Center, Sun Yat-sen University, Guangzhou 510060, People’s Republic of China \\ ${ }^{3}$ Department of Medical Oncology, Cancer Center, Sun Yat-Sen University, Guangzhou 510060, People's Republic of China \\ ${ }^{4}$ Chengdu Kanghong Biotechnology Co., Ltd, Chengdu 610036, People's Republic of China \\ "These authors equally contributed
}

Received: August 14, 2015; Accepted: December 07, 2015; Published: December 10, 2015

*Corresponding author: Su Li, Department of Clinical Research Center, Cancer Center, Sun Yat-sen University, Guangzhou 510060, People's Republic of China. Tel: +862087343145; E-mail: lisu@sysucc.org.cn

\begin{abstract}
KH903 is a novel antiangiogenic drug for cancer therapy. Its pharmacokinetic study in cancer patients is therefore very important for choosing doses and dosing intervals in clinical application. A selective, sensitive, and rapid Enzyme-Linked Immunosorbent Assay (ELISA) method was developed and validated here for the determination of KH903 in patient serum. This method is specific enough for the detection of KH903 in serum samples. Nonlinear calibration curves were obtained for KH903 at a concentration range of $0.781-50 \mathrm{ng} / \mathrm{ml}(r>0.999)$. The intra-batch accuracy ranged from $83.4 \%$ to $94.5 \%$, and the inter-batch accuracy varied from $88.8 \%$ to $91.7 \%$. Stability tests showed that KH903 was stable throughout the analytical procedure. This study is the first to utilize the ELISA method for the pharmacokinetic study of KH903 in six cancer patients who had received a single dose of KH903 (4.0 mg/ kg) administered intravenously. The results address the pharmacokinetic profile of KH903 in cancer patients in detail and evaluate the binding efficacy of KH903 on VEGF in patients to provide guidance for the regimen of KH903 in phase I clinical trials.
\end{abstract}

Keywords: KH903; Enzyme-linked Immunosorbent Assay; Pharmacokinetic study; Antiangiogenic drug; VEGF

\section{Introduction}

Angiogenesis is an important hallmark of cancer during the multistep development of tumors [1]. The tumor-associated neovasculature generated in the process of angiogenesis, from which tumors fulfill the requirements of nutrients and oxygen as well as the ability to excrete metabolic wastes. It's established that tumors achieve vasculature by endothelial cell sprouting [2], postnatal vasculogenesis [3], intussusceptive growth [4,5], preexisting vessel cooption [6-9], or glomeruloid angiogenesis [10]. Vascular Endothelial Growth Factor (VEGF) is known as a key mediator in some or all of the neovasculature processes, therefore, VEGF is considered as an important target for antiangiogenic drug development [11-14].

Blockage of VEGF has been considered to be useful against a wide variety of tumors $[13,15,16]$. The first attempt to validate this particular approach were taken by a humanized monoclonal antibody bevacizumab, which yielded therapeutic effect in a clinical trial in patients with renal cell carcinoma $[17,18]$. Several anti-VEGF drugs have since been approved or are proceeding in clinical trials $[19,20]$. KH903, also known as Conbercept, is a novel recombinant VEGF receptor-antibody fusion protein (Figure 1), which was demonstrated to exhibit an inhibitory effect on the VEGF-mediated proliferation and migration of human umbilical vein endothelial cells, and on the patient-derived tumor tissue xenograft model of gastric carcinoma through an antiangiogenic mechanism [21,22].

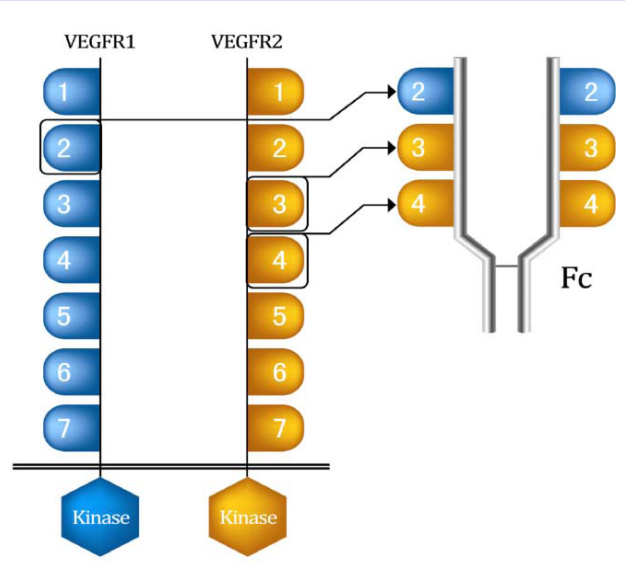

Figure 1: Structure of KH903. KH903 is a recombinant VEGF receptor-antibody fusion protein, which contains the extracellular domain 2 of VEGF receptor 1 and the extracellular domains 3 and 4 of VEGF receptor 2, fused to the Fc portion of human immunoglobulin G1. 
KH903 was approved for phase I clinical trial as a new medicine in 2009 by the China Food and Drug Administration (CFDA). Clinical trial of a novel drug requires reliable data's about its pharmacokinetic properties, the method for measuring levels of the drug in biological samples is thus necessary. KH903 is a novel antiangiogenic drug, few methods have been developed for its quantification. Therefore, a sufficient specific and sensitive Enzyme-Linked Immunosorbent Assay (ELISA) was developed to determine the serum concentration of KH903 in cancer patients in a pharmacokinetic study. The method was validated for its specificity, sensitivity, accuracy, precision, stability, and dilution integrity, and the data established the method as a high-throughput and reliable bioanalytical assay. Moreover, VEGF itself has been considered as a candidate biomarker for guiding the application of anti-VEGF therapies, we thus have measured the serum unbound VEGF in cancer patients after the administration of KH903.

\section{Materials and Methods}

\section{Experimental chemicals}

KH903 was provided by Chengdu Kanghong Biological Technology Co., Ltd (Sichuan, China). Recombinant human VEGF (rhVEGF), human VEGF ELISA kits, and tetramethylbenzidine (TMB) were purchased from R\&D (Minneapolis, MN, USA). Anti-hIgG Fc-HRP was purchased from BETHYL (Montgomery, TX, USA). Fetal Bovine Serum (FBS) was purchased from PAA (Karlsruhe, Germany). Tween-20 and DMSO were purchased from Sigma (St. Louis, MO, USA). De-ionized water was obtained by a Milli- $Q$ analytical deionization system (Millipore, Bedford, MA, USA). Freshly obtained, drug-free human serum was collected from healthy people and stored at $-80^{\circ} \mathrm{C}$ before used.

\section{ELISA procedure for $\mathrm{KH} 903$}

Polystyrene 96-place microwell plates were treated with $100 \mu \mathrm{L} /$ well of a $0.5 \mu \mathrm{g} / \mathrm{ml}$ solution of rhVEGF in carbonate buffer and incubated for $16 \mathrm{~h}$ at $25^{\circ} \mathrm{C}$. Wells were then washed three times with washing buffer ( $5 \mathrm{mM}$ imidazole buffer, $0.02 \%$ Tween-20, pH 7.6), and unbound sites were blocked with 280 $\mu \mathrm{L} /$ well of $40 \%(\mathrm{v} / \mathrm{v}) \mathrm{FBS}$ in carbonate buffer for $2 \mathrm{~h}$ at $37^{\circ} \mathrm{C}$. The calibrator and Quality Control (QC) consisted of the normal serum spiked with KH903. The samples were diluted 5, 10, or 20 fold by drug-free human serum prior to the ELISA procedure; their final concentrations are required to multiply by the serum dilution fold. After the wells were washed and blotted dry, the calibrator, quality control, and the samples diluted for 80 fold with dilution buffer (PBS, 10\% FBS, 0.05\% Tween-20, 15\% DMSO) were added and incubated $100 \mu \mathrm{L} /$ well for $1 \mathrm{~h}$ at $37^{\circ} \mathrm{C}$. The wells were washed three times, and $100 \mu \mathrm{L} /$ well of antihIgG Fc-HRP (1:20000) were added. After $1 \mathrm{~h}$ at $37^{\circ} \mathrm{C}$, the wells were washed and blotted dry, then added $100 \mu \mathrm{L} /$ well of TMB substrate solution and incubated at room temperature for 10 min. The reaction was stopped by adding $50 \mu \mathrm{L} /$ well $2 \mathrm{M} \mathrm{H}_{2} \mathrm{SO}_{4}$, and the absorbance was measured at $450 \mathrm{~nm}$ on Molecular DEVICES Versa Max microplate Reader (Sunnyvale, CA, USA). Data corresponding to the averages of double well replicates were processed using SoftMax Pro 5.4.1 (Sunnyvale, CA, USA).

\section{Specificity determination}

The specificity was tested against the responses toward KH903. Each concentration of the compounds was tested in double well replicates. The VEGF-KH903 solution was obtained by incubating $\mathrm{KH} 903$ and VEGF together in $37^{\circ} \mathrm{C}$ for $45 \mathrm{~min}$. KH903, VEGF, and VEGF-KH903 were spiked in dilution buffer, respectively, and KH903 spiked in dilution buffer with $1.25 \%$ serum $(\mathrm{v} / \mathrm{v})$ was also prepared for the determination. The specificity was evaluated according to the OD values.

\section{Method validation}

KH903 was validated for an ELISA assay. The Lower Limit of Quantification (LLOQ), the Upper Limit of Quantification (ULOQ), calibration curve, accuracy, precision, recovery, and stability were evaluated during method validation. LLOQ and ULOQ were conducted as the minimal and maximal concentration on the calibration curve in six different batches, on which both precision and accuracy were $\leq 25 \%$. The range of the calibration curve was $0.781 \sim 50 \mathrm{ng} / \mathrm{mL}$. Calibration curve equations were calculated using four-parameter logistic nonlinear regression model, from which the correlation coefficient $(r>0.99)$ was considered satisfactory. Precision and accuracy were assessed by the analyses covering the range of the calibration curve, in which the criteria for acceptability is defined as an accuracy $\pm 20 \%$ standard deviation (SD) from the nominal values and a precision of $\pm 20 \%$ Relative Standard Deviation (RSD). Intra-batch accuracy and precision were evaluated by analyzing three levels of QC samples with six duplicated levels per concentration on the same day. The inter-batch accuracy and precision were assessed over three days. The recovery of KH903 was determined by calculating the ratio of the OD value of KH903 spiked in dilution buffer with $1.25 \%$ serum $(\mathrm{v} / \mathrm{v})$ against the value spiked in dilution buffer at the same concentration. The stability of KH903 was compared to the nominal level of KH903 to determine whether KH903 was stable in different conditions or treatments. If the calculated concentration of KH903 was less than the nominal concentration by $>20 \%$, the analyte was considered to be unstable. The stability of KH903 spiked in serum was evaluated by exposing them at room temperature for $4 \mathrm{~h}$ and then subjecting them to the analytical procedure. KH903 maintained at $-80^{\circ} \mathrm{C}$ for 12 months was evaluated by comparing the post-freeze measured KH903 concentration with the initial concentration added to the sample. The freeze-thaw stability of the samples was assessed over three freeze-thaw cycles by thawing samples at room temperature, refreezing them for $24 \mathrm{~h}$ at $-80^{\circ} \mathrm{C}$ and then analyzing them. Dilution integrity was evaluated by diluting 5, 10, 20 folds of the dilution QC samples with drug-free human serum prior to the ELISA procedure.

\section{Applications}

Six patients with gastric, colonic, nasopharyngeal, or thymic carcinoma were enrolled at the Cancer Center, Sun Yat-sen University. The patients were four males and two female, ranging in age from 34 to 65 years. All patients provided written informed consent prior to participation. The patients were infused (i.v. administration) with KH903 (4.0 mg/ kg) for $90 \mathrm{~min}$. Blood 
samples were obtained before the infusion, and $0.75,1.5,2,6,12$, $24,48,72,120,168,216$, and $264 \mathrm{~h}$ after the infusion began. In addition, blood samples were obtained before the infusion, and $1.5,24,72,120,168,216,264$, and $312 \mathrm{~h}$ after the infusion began for the unbound VEGF determination using ELISA kits. The blood samples were centrifuged at $3000 \mathrm{rpm} / \mathrm{min}$ for $10 \mathrm{~min}$, and the serum was stored at $-80^{\circ} \mathrm{C}$ until the analysis was conducted. This study was approved by the Human Subjects Review Committees of the University of Sun Yat-sen University Cancer Center and conducted according to the Declaration of Helsinki.

Non-compartmental pharmacokinetic parameters were calculated using WinNonlin (Version 5.0, PUMCH Clinical Pharmacology Research Center). The maximum serum concentration $\left(\mathrm{C}_{\max }\right)$ and time to reach it $\left(\mathrm{T}_{\max }\right)$ were determined directly from the data. The terminal-phase rate constant $(\lambda z)$ was calculated as the negative slope of the log-linear terminal portion of the serum concentration-time curve using linear regression with at least four last concentration-time points. The terminalphase half-life $\left(t_{1 / 2}\right)$ was calculated as $0.693 / \lambda z$. The area under the curve from time zero to the last observed time $\left(\mathrm{AUC}_{0-\mathrm{f}}\right)$ was calculated by the linear trapezoidal rule for ascending data points. The total area under the curve $\left(\mathrm{AUC}_{0-\infty}\right)$ was calculated as $\mathrm{AUC}_{0-\mathrm{t}}+\mathrm{Ct} / \lambda \mathrm{z}$, where $\mathrm{Ct}$ is the last measurable concentration. The apparent volume of distribution associated with the terminal phase $(\mathrm{Vz})$ was calculated as $\mathrm{Vz}=\mathrm{CL} / \lambda \mathrm{z}$, and the apparent total body clearance (CL) was calculated as $\mathrm{CL}=$ dose/AUC.

\section{Results and Discussion}

\section{Optimization of ELISA conditions}

To improve the ELISA performance, several factors (concentration of rhVEGF, time for coating 96 well plates with rhVEGF, and dilution of anti-hIgG Fc-HRP) were studied. The value of the highest absorbance was selected as the parameter for evaluation of the assay. We found that $0.5 \mu \mathrm{g} / \mathrm{ml}$ rhVEGF coating with 96 well plates for $16 \mathrm{~h}$ was more satisfactory than other conditions in the sensitivity and stability of the assay. Different concentrations of anti-hIgG Fc-HRP were examined in the optimization procedure, and the final dilution was established as 1:20000. In the pre-experiment, we found an obvious intervention in the absorbance assay with human serum, which is probably related to the non-specific binding with IgG [23]. A series of dilution $(1: 20,1: 40,1: 60,1: 80,1: 100,1: 200)$ with dilution buffer were investigated in the serum samples of KH903, the optimal effect of eliminating non-specific binding was achieved in 1:80 dilution (data not shown), and this content was chosen for the sample preparation.

\section{Specificity investigation}

Based on the mechanism of the reaction, VEGF-KH903 and endogenous VEGF are the possible interferents toward the KH903 determination. In order to investigate the specificity of the response to KH903 in this method, KH903, VEGF, VEGF-KH903, and KH903 spiked in $1.25 \%$ serum were evaluated according to the OD values. As shown in Table 1, the OD values of KH903 were positively related with the corresponding concentrations, but the OD values of VEGF were not changed along with the reaction concentrations. The measured OD values of VEGF-KH903 were lower than the equivalent theoretical levels of KH903. The high level of VEGF-KH903 exhibited increased OD values, speculating that OD values depend on the level of the unbound KH903 but not the bound VEGF-KH903. Dilution buffer spiked in 1.25\% serum shown higher background in the absorbance test, and the KH903 samples spiked in $1.25 \%$ serum also exhibited higher absorbance; these results are probably due to the non-specific binding with IgG in human serum, which is $8-17 \mathrm{mg} / \mathrm{mL}$ in adult blood [24]. Therefore, the present method is specific enough for the detection of KH903 in serum samples.

\section{Calibration curve, LLOQ, and ULOQ}

The calibration curves were determined from the absorbance versus concentration in human serum, and the equations were calculated using four-parameter logistic nonlinear regression model. Human serum KH903 was detected by the method, overing the range tested $(0.781-50 \mathrm{ng} / \mathrm{mL})$. The typical equation for the calibration curve for KH903 was $\mathrm{y}=-2.773$ / $\left(1+(\mathrm{x} / 14.4)^{1.22}\right)+2.88(r=0.999)$. The LLOQ and ULOQ in this study were $0.781 \mathrm{ng} / \mathrm{mL}$ and $50 \mathrm{ng} / \mathrm{mL}$, respectively, and the precision of repeat injections was $<25 \%$. All the actual concentrations of the unknown biological samples equal to the calculated concentrations multiplied by 80 .

\section{Accuracy, precision, and recovery}

The accuracy and precision of the method were determined by analyzing QC samples at three concentrations in six replicates. As shown in Table 2, the intra-batch accuracy ranged from $83.4 \%$ to $94.5 \%$ at three concentrations, with precisions between $3.9 \%$ and $4.6 \%$. The inter-batch accuracy varied from $88.8 \%$ to $91.7 \%$, with precisions ranging from $8.6 \%$ to $12.4 \%$. Thus, the present

\begin{tabular}{|c|c|c|}
\hline Group & Concentration (ng/mL) & OD Value \\
\hline \multirow{4}{*}{ KH903 } & 50 & 1.427 \\
\hline & 5 & 0.386 \\
\hline & 0.5 & 0.107 \\
\hline & Buffer & 0.048 \\
\hline \multirow{4}{*}{ VEGF } & 500 & 0.051 \\
\hline & 50 & 0.051 \\
\hline & 5 & 0.052 \\
\hline & Buffer & 0.046 \\
\hline \multirow{4}{*}{ KH903+VEGF } & $286 / 8.8$ & 1.712 \\
\hline & $28.6 / 8.8$ & 0.869 \\
\hline & $2.86 / 8.8$ & 0.081 \\
\hline & Buffer & 0.060 \\
\hline \multirow{4}{*}{ KH903+serum } & 50 & 1.479 \\
\hline & 5 & 0.581 \\
\hline & 0.5 & 0.293 \\
\hline & Buffer with serum & 0.120 \\
\hline
\end{tabular}


method has satisfactory accuracy, precision and reproducibility The recoveries from QC samples at low, intermediate and high concentrations ranged from $85.3 \%$ to $92.9 \%$ at three tested concentrations.

\section{Stability of KH903}

The results from the stability tests are presented in Table 3, the accuracy ranged from $81.3 \%$ to $112.0 \%$ at the three dilution folds, with precisions between $3.4 \%$ and $13.1 \%$. The data demonstrate a good stability of KH903 throughout the steps of the determination. The method is therefore applicable to routine analyses.

\section{Dilution integrity of KH903}

5, 10, 20 folds of the dilution QC samples with drug-free human serum were carried out to evaluate the reproducibility and stability in dilution procedure. As shown in Table 4, the accuracy ranged from $86.8 \%$ to $101.6 \%$ at the three dilution folds, with precisions between $5.2 \%$ and $8.5 \%$, demonstrating a well reproducible dilution procedure in the present study.

\section{Analysis of patient samples}

The ocular pharmacokinetics profile of Conbercept in rabbits has been evaluated [25], but its systemic pharmacokinetics in patient is still unknown. The validated ELISA method described in our study was successfully applied to a pharmacokinetic study in 6 cancer patients following i.v. administration of $4.0 \mathrm{mg} / \mathrm{kg}$ KH903. A mean plasma concentration-time curve of a single dose of KH903 is shown in Figure 2. This result revealed that our method was sufficiently sensitive to determine the KH903 concentration in the serum of patients. The parameters of the pharmacokinetic analysis are shown in Table 5. The half-life of drug elimination at the terminal phase $\left(t_{1 / 2}\right)$ was $29.90 \pm 15.33 \mathrm{~h}$, the volume of distribution $\left(\mathrm{V}_{\mathrm{d}}\right)$ of KH903 was $17.19 \pm 6.93 \mathrm{~L}$, and the total clearance $(\mathrm{CL})$ was $0.39 \pm 0.05 \mathrm{~L} / \mathrm{h}$. All the parameters indicated KH903 good pharmacokinetic profile in cancer patients and this evidence can be guidance for the regimen of KH903 in phase I clinical trials.

KH903 is a novel fusion protein that combines ligand binding elements from the extracellular domains of VEGF receptors and the Fc portion of IgG1, and is able to bind to VEGF [21]. It's demonstrated that blockage of VEGF significantly inhibited the growth of a non-small cell lung cancer cell line, and markedly decreased the vessel density of the tumor [26]. Treatment with antiangiogenic drugs can transiently reverse the abnormalities of tumor vessels, thereby providing a window of opportunity for improving drug delivery and enhancing sensitivity to conventional chemotherapy and radiation treatment [27-29]. The serum unbound VEGF concentration is therefore investigated here to illustrate the binding efficacy of KH903 on serum VEGF. As shown in Figure 3, the serum levels of unbound VEGF of each patient decreased rapidly to below the detection limit after

Table 2: Accuracy, precision and recovery of KH903.

\begin{tabular}{|c|c|c|c|c|c|}
\hline \multirow{2}{*}{$\begin{array}{c}\text { Concentration } \\
\text { (ng/ mL) }\end{array}$} & \multicolumn{2}{|c|}{ Intra-batch (n= 6) } & \multicolumn{2}{|c|}{ Inter-batch (n= 3) } \\
\hline 2 & Accuracy (\%) & RSD (\%) & Accuracy (\%) & RSD (\%) \\
\hline 25 & 83.4 & 4.0 & 91.7 & 12.4 \\
\hline 40 & 88.6 & 3.9 & 91.4 & 9.1 \\
\hline
\end{tabular}

Table 3: Stability of KH903 in different storage conditions.

\begin{tabular}{|c|c|c|c|}
\hline Storage conditions & Concentration (ng/mL) & Accuracy $(\%)(n=6)$ & RSD (\%) \\
\hline \multirow{3}{*}{ Freeze-thaw three cycles } & 2 & 110.2 & 5.7 \\
\hline & 25 & 112.0 & 5.5 \\
\hline & 40 & 99.2 & 9.7 \\
\hline \multirow{3}{*}{$-80^{\circ} \mathrm{C}$ for 12 months } & 2 & 81.3 & 5.9 \\
\hline & 25 & 86.7 & 5.6 \\
\hline & 40 & 83.1 & 7.6 \\
\hline \multirow{3}{*}{ Room temperature for $4 \mathrm{~h}$} & 2 & 96.6 & 13.1 \\
\hline & 25 & 94.7 & 3.4 \\
\hline & 40 & 85.2 & 4.0 \\
\hline
\end{tabular}

Table 4: Dilution integrity of KH903.

\begin{tabular}{|c|c|c|c|}
\hline Table 4: Dilution integrity of KH903. \\
\hline Dilution Factor & Analyte Concentration (ng/ mL) & Calculated Concentration (ng/ mL) & $\begin{array}{c}\text { Accuracy (\%) } \\
\text { (n = 6) }\end{array}$ \\
\hline 20-fold & 25 & 25.4 & 101.6 \\
\hline 10-fold & 25 & 23.9 & 95.7 \\
\hline 5-fold & 25 & 21.7 & 5.2 \\
\hline
\end{tabular}




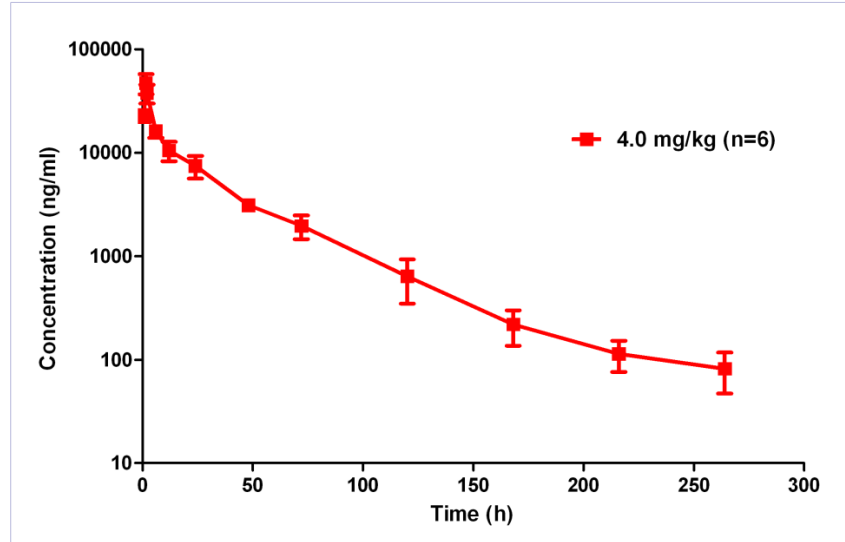

Figure 2: Mean serum concentration-time curve of KH903 after 4.0 $\mathrm{mg} / \mathrm{kg}$ single i.v. administration to cancer patients $(\mathrm{n}=6)$.

Table 5: Non-compartmental pharmacokinetic parameters of KH903 in the cancer patients after single i.v. dose of $4.0 \mathrm{mg} / \mathrm{kg} \mathrm{KH903}(\mathrm{n}=6)$.

\begin{tabular}{|c|c|}
\hline Parameters & Values \\
\hline Tmax $(\mathrm{h})^{\mathrm{a}}$ & $1.50 \pm 0.00$ \\
\hline $\mathrm{Cmax}(\mu \mathrm{g} / \mathrm{mL})^{\mathrm{b}}$ & $47.22 \pm 6.24$ \\
\hline $\mathrm{t} 1 / 2(\mathrm{~h})^{\mathrm{c}}$ & $29.90 \pm 15.33$ \\
\hline $\begin{array}{c}\mathrm{AUC}_{0-\mathrm{t}}^{\mathrm{d}} \\
\left(\mu \mathrm{g} \cdot \mathrm{mL}^{-1} \cdot \mathrm{h}\right)\end{array}$ & $628.83 \pm 75.15$ \\
\hline $\begin{array}{c}\mathrm{AUC}_{0-0}{ }^{\mathrm{e}} \\
\left(\mu \mathrm{g} \cdot \mathrm{mL}^{-1} \cdot \mathrm{h}\right)\end{array}$ & $633.48 \pm 75.74$ \\
\hline $\mathrm{V}_{\mathrm{d}}(\mathrm{L})^{\mathrm{f}}$ & $17.19 \pm 6.93$ \\
\hline $\mathrm{CL}(\mathrm{L} / \mathrm{h})^{\mathrm{g}}$ & $0.39 \pm 0.05$ \\
\hline $\mathrm{MRT}(\mathrm{h})^{\mathrm{h}}$ & $30.59 \pm 6.85$ \\
\hline
\end{tabular}

Values are means + SD.

${ }^{a}$ Tmax: Time to maximum concentration; ${ }^{b} \mathrm{C}$ max: Maximum plasma concentration; ${ }^{c} \mathrm{t}_{1 / 2}$ : Half-life of elimination; ${ }^{\mathrm{d}} \mathrm{AUC}_{0 . \mathrm{t}}$ : Area under the concentration-time curve from zero to last quantifiable time; ${ }^{\mathrm{e}} \mathrm{AUC}_{0-\infty}$ : Area under the concentration-time curve extrapolated to infinity; ${ }^{\mathrm{f}-\infty} \mathrm{V}_{\mathrm{d}}$ : Volume of distribution; ${ }^{\mathrm{g}} \mathrm{CL}$ : Total clearance; ${ }^{\mathrm{h}} \mathrm{MRT}$ : Mean residence time.

intravenous infusion KH903 indicated that KH903 indeed blocked the VEGF by the specific binding. Accompanying the decline of the KH903, the level of unbound VEGF gradually increased to more than $100 \mathrm{pg} / \mathrm{mL}$, suggesting that the reasonable dosing interval of KH903 should be ascertained in future studies for obtaining the persistent inhibition in VEGF.

In conclusion, a selective, sensitive, and rapid ELISA method for measuring KH903 in human serum is described. The present description is the first to utilize the ELISA method for the pharmacokinetic study of KH903 given by injection to cancer patients. The results address the pharmacokinetic profile of KH903 in cancer patients in detail and evaluate the binding efficacy of KH903 on VEGF in patients to provide guidance for the regimen of KH903 in phase I clinical trials.

\section{Acknowledgements}

This study was supported by the research grant from

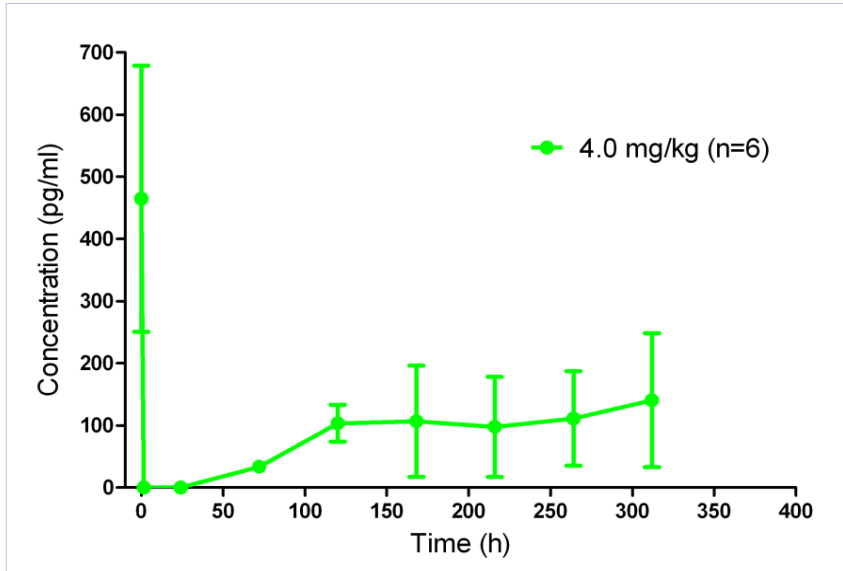

Figure 3: Mean serum concentration-time curve of unbound VEGF after $4.0 \mathrm{mg} / \mathrm{kg}$ single i.v. administration of KH903 to cancer patients $(n=6)$.

the Natural Science Foundation of Guangdong province (no. S2013010016452) and Chengdu Kanghong Biotechnology Co., Ltd.

\section{Declarations}

There is no conflict of interests among the authors regarding the publication of this article. KH903 in the clinical trial was approved for phase I clinical trial as a new medicine in 2009 by the China Food and Drug Administration (CFDA), and the trial was approved by the Human Subjects Review Committees of the University of Sun Yat-sen University Cancer Center and conducted according to the Declaration of Helsinki.

\section{References}

1. Hanahan D and Weinberg RA. Hallmarks of cancer: the next generation. Cell. 2011;144(5):646-74. doi: 10.1016/j.cell.2011.02.013.

2. Lai Y, Liu XH, Zeng Y, Zhang Y, Shen Y, Liu Y. Interleukin-8 induces the endothelial cell migration through the Rac1/RhoA-p38MAPK pathway. Eur Rev Med Pharmacol Sci. 2012;16(5):630-8.

3. 3. Sundberg C, Nagy JA, Brown LF, Feng D, Eckelhoefer IA, Manseau EJ. Glomeruloid microvascular proliferation follows adenoviral vascular permeability factor/vascular endothelial growth factor-164 gene delivery. Am J Pathol. 2001;158(3):1145-60.

4. Kurz H, Burri PH, Djonov VG. Angiogenesis and vascular remodeling by intussusception: from form to function. News Physiol Sci. 2003;18:6570.

5. Osawa M, Masuda M, Kusano K, Fujiwara K. Evidence for a role of platelet endothelial cell adhesion molecule-1 in endothelial cell mechanosignal transduction: is it a mechanoresponsive molecule? J Cell Biol. 2002;158(4):773-85.

6. Holash J, Maisonpierre PC, Compton D, Boland P, Alexander CR, Zagzag $\mathrm{D}$, et al. Vessel cooption, regression, and growth in tumors mediated by angiopoietins and VEGF. Science. 1999;284(5422):1994-8.

7. Döme B, Paku S, Somlai B, Tímár J. Vascularization of cutaneous melanoma involves vessel co-option and has clinical significance. J Pathol. 2002;197(3):355-62.

8. Asai T. Nanoparticle-mediated delivery of anticancer agents to tumor 
angiogenic vessels. Biol Pharm Bull. 2012;35(11):1855-61.

9. Paku S, Kopper L, Nagy P. Development of the vasculature in 'pushingtype' liver metastases of an experimental colorectal cancer. Int Cancer. 2005;115(6):893-902.

10. Asahara T, Kawamoto A. Endothelial progenitor cells for postnatal vasculogenesis. Am J Physiol Cell Physiol. 2004;287(3):C572-9.

11. Hicklin DJ, Ellis LM. Role of the vascular endothelial growth factor pathway in tumor growth and angiogenesis. J Clin Oncol. 2005;23(5):1011-27.

12. Ferrara N, Gerber HP, LeCouter J. The biology of VEGF and its receptors. Nat Med. 2003;9(6):669-76.

13. Teng LS, Jin KT, He KF, Wang HH, Cao J, Yu DC. Advances in combination of antiangiogenic agents targeting VEGF-binding and conventional chemotherapy and radiation for cancer treatment. J Chin Med Assoc. 2010;73(6):281-8. doi: 10.1016/S1726-4901(10)70062-9.

14. Wang K, Yang R, Xu J, Zhang Y, Zhu L, Lin J, et al. Development of an AlphaScreen-based high-throughput screening assay for inhibitors of human vascular endothelial growth factor receptor-3. J Immunoassay Immunochem. 2011;32(3):219-32. doi $10.1080 / 15321819.2011 .559296$.

15. Teng LS, Jin KT, He KF, Zhang J, Wang HH, Cao J. Clinical applications of VEGF-trap (aflibercept in cancer treatment. J Chin Med Assoc. 2010;73(9):449-56. doi: 10.1016/S1726-4901(10)70097-6.

16. Collet G, Lamerant-Fayel N, Tertil M, El Hafny-Rahbi B, Stepniewski J, Guichard A, et al. Hypoxia-regulated overexpression of soluble VEGFR2 controls angiogenesis and inhibits tumor growth. Mol Cancer Ther. 2014;13(1):165-78. doi: 10.1158/1535-7163.MCT-13-0637

17. Yang JC, Haworth L, Sherry RM, Hwu P, Schwartzentruber DJ, Topalian $\mathrm{SL}$, et al. A randomized trial of bevacizumab, an anti-vascular endothelial growth factor antibody, for metastatic renal cancer. N Engl J Med. 2003;349(5):427-34.

18. Yang JC. Bevacizumab for patients with metastatic renal cancer: an update. Clin Cancer Res. 2004;10(18 Pt 2):6367S-70S.

19. Jubb AM, Oates AJ, Holden S, Koeppen H. Predicting benefit from antiangiogenic agents in malignancy. Nat Rev Cancer. 2006;6(8):626-35.
20. Quesada AR, Muñoz-Chápuli R, Medina MA. Anti-angiogenic drugs: from bench to clinical trials. Med Res Rev. 2006;26(4):483-530.

21. Wu Z, Zhou P, Li X, Wang H, Luo D, Qiao H, et al. Structural characterization of a recombinant fusion protein by instrumental analysis and molecular modeling. PLoS One. 2013;8(3):e57642. doi: 10.1371/journal.pone.0057642.

22. Jin K, Lan H, Cao F, Xu Z, Han N, Li G, et al. Antitumor effect of FP3 in a patient derived tumor tissue xenograft model of gastric carcinoma through an antiangiogenic mechanism. Oncol Lett. 2012;3(5):10521058.

23. Xiao Y, Isaacs SN. Enzyme-linked immunosorbent assay (ELISA and blocking with bovine serum albumin (BSA-not all BSAs are alike. J Immunol Methods. 2012;384(1-2):148-51. doi: 10.1016/j. jim.2012.06.009.

24.Stebler C, Tichelli A, Dazzi H, Wernli M, Gratwohl A, Speck B. Immunoglobulin deficiency after repeated plasmapheresis. Schweiz Med Wochenschr. 1991;121(5):145-9.

25.Li H, Lei N, Zhang M, Li Y, Xiao H, Hao X. Pharmacokinetics of a long-lasting anti-VEGF fusion protein in rabbit. Exp Eye Res. 2012;97(1):154-9. doi: 10.1016/j.exer.2011.09.002.

26. Jin $\mathrm{K}$, He K, Teng F, Li G, Wang H, Han N, et al. FP3: a novel VEGF blocker with anti-angiogenic effects in vitro and antitumour effects in vivo. Clin Transl Oncol. 2011;13(12):878-84. doi: 10.1007/s12094011-0749-z.

27. Shalaby MA, Hampson L, Oliver A, Hampson I. Plexin D1: new potential biomarker for cervical cancer. J Immunoassay Immunochem. 2012;33(3):223-33. doi: 10.1080/15321819.2011.634472.

28. Willett CG, Boucher Y, di Tomaso E, Duda DG, Munn LL, Tong RT, et al. Direct evidence that the VEGF-specific antibody bevacizumab has antivascular effects in human rectal cancer. Nat Med. 2004;10(2):1457.

29. Baffert F, Le T, Sennino B, Thurston G, Kuo CJ, Hu-Lowe D, et al. Cellular changes in normal blood capillaries undergoing regression after inhibition of VEGF signaling. Am J Physiol Heart Circ Physiol. 2006;290(2):H547-59. 